\title{
Introduction to "On the use of Markov chain Monte Carlo methods for the sampling of mixture models" by R. Douc, F. Maire, J. Olsson
}

\author{
Stefano Peluso
}

Published online: 29 October 2014

(C) Springer Science+Business Media New York 2014

The Authors compare, theoretically and by simulations, various algorithms for sampling mixture models. The starting point is the algorithm of Carlin and Chib (1995), which augments the parameter space through the involvement of socalled pseudo priors or linking functions. Carlin and Chib (1995) originally introduced their algorithm (from now on, the $\mathrm{CC}$ algorithm) with the purpose of overcoming some limitations implicit in more standard MCMC approaches, for instance in the case of models with parameter space of varying size. In the development of the present paper, the $\mathrm{CC}$ algorithm is implemented in the context of mixture models of fixed size, with the aim of improving the mixing of a standard Gibbs sampler, whose performance is negatively affected by the high correlation between mixture weights and components.

The first contribution is the generalization of the $\mathrm{CC}$ algorithm along two directions: the first one involves the substitution of the Gibbs step by a Metropolis step, in order to deal with a framework in which the specific distribution of the mixture component cannot be directly sampled (MCC algorithm). This is a fairly standard practice and typically the resulting algorithms are named "Metropolis within Gibbs". The second, more substantial, generalization is motivated by computational considerations that lead the authors to replace the Gibbs step mentioned above with a deterministic assignment (FCC algorithm). The new algorithms are justified theoretically and compared in two examples. The second example is particularly relevant in showing that the proposed FCC algorithm can have competing inference performances with a considerable saving of CPU time and a negligible loss of pre-

S. Peluso $(\varangle)$

Swiss Finance Institute, University of Lugano, Via Giuseppe Buffi 13, 6904 Lugano, Switzerland

e-mail: stefano.peluso@usi.ch cision. This suggests an escamotage that, in principle, could be applied to other MCMC procedures with high computational complexity, as already done in the grouped independence metropolis hastings (GIMH) algorithm of Beaumont (2003), as also mentioned by the Authors.

The second contribution of the paper is the theoretical analysis of the proposed algorithms, that are nonreversible, exploiting the framework of inhomogeneous Markov chains developed in the forthcoming paper of Maire et al. (2014). Asymptotic results on Markov chains are easier to establish for reversible Markov chains, but reversibility is not a necessary condition for having the correct stationary distribution. Furthermore, nonreversible Markov chains are often built on purpose, in order to improve asymptotic properties of the resulting MCMC estimators (see, for instance Mira and Geyer 1999, 2000; Diaconis et al. 2000 and references therein). The framework presented suggests a relatively easy and general procedure that can be applied to analyze other nonreversible homogeneous Markov Chains obtained by composing reversible Markov kernels.

Assessing model adequacy and selecting the best model was the main aim of the $\mathrm{CC}$ algorithm, so Bayes factor computation is a potential field of application to successfully test the proposed algorithms. By introducing the model indicator as unknown parameter, Bayes factors can be computed without attempting to calculate the marginal likelihood. However, it is necessary to specify all the competing models, a task that may not always be possible. In this setting the algorithms proposed can provide suggestions for a computationally more efficient alternative to the calculation of the marginal likelihood, along the lines of Chib (1995) and Chib and Jeliazkov (2001). The freezing idea behind the FCC sampler seems also suitable for application to the method introduced in Stephens (2000) for the analysis of finite mixture distributions with an unknown number of components. 


\section{References}

Beaumont, M.: Estimation of population growth or decline in genetically monitored populations. Genetics 164, 1139-1160 (2003)

Carlin, B., Chib, S.: Bayesian model choice via Markov chain Monte Carlo Methods. J. Royal Stat. Soc. Ser. B 57, 473-484 (1995)

Chib, S.: Marginal likelihood from the Gibbs output. J. Am. Stat. Assoc. 90, 1313-1321 (1995)

Chib, S., Jeliazkov, I.: Marginal likelihood from the MetropolisHastings output. J. Am. Stat. Assoc. 96, 270-281 (2001)

Diaconis, P., Holmes, S., Neal, R.: Analysis of a nonreversible Markov chain sampler. Ann. Appl. Probab. 10, 726-752 (2000)
Maire, F., Douc, R., Olsson, J.: Comparison of asymptotic variances of inhomogeneous Markov chains Monte Carlo Methods. Ann. Stat., forthcoming (2014)

Mira, A., Geyer, C.: Ordering Monte Carlo Markov Chains. Technical Report, Universit degli Studi dell'Insubria. http://eco.uninsubria. it/webdocenti/amira/papers/tr632.ps (1999)

Mira, A., Geyer, C.: On nonreversible Markov chains. In Fields Instit. Commun. Monte Carlo Methods 26, 93-108 (2000)

Stephens, M.: Bayesian analysis of mixture models with an unknown number of components -An alternative to reversible jump methods. Ann. Stat. 28, 40-74 (2000) 